\title{
SwissDRG: komplexe Prozeduren nachvollziehbar dokumentieren
}

\author{
Die Dokumentation ärztlicher Diagnostik- und Therapiemassnahmen gewinnt mit \\ der Einführung von SwissDRG zunehmend an Bedeutung. Nur dokumentierte Leis- \\ tungen werden von den Kodierfachpersonen kodiert und von den Kostenträgern \\ bezahlt. Um die Ärzteschaft in der Dokumentation komplexer Leistungen zu unter- \\ stützen, stellt die FMH 35 Dokumentations-Muster auf ihrer Website zur Verfügung.
}

Petra Ingenpass

Stellvertretende Leiterin Bereich SwissDRG der FMH
Korrespondenz: Dr. med. Petra Ingenpass Tarifdienst FMH Froburgstrasse 15 CH-4600 Olten Tel. 0313591111 swissdrg@fmh.ch
Mit der Prozedurenklassifikation CHOP 2011 steht seit dem 1. Januar 2011 ein umfangreicher Katalog zur Erfassung von im Spital erbrachten Leistungen zur Verfügung. Diese detaillierte Darstellung ist auf das Engagement der Fachgesellschaften zurückzuführen, haben sie doch in den Antragsverfahren der letzten Jahre nahezu 2000 Anträge mit Vorschlägen zur Differenzierung bereits bestehender Prozeduren oder zur Neuaufnahme bisher nicht abbildbarer Behandlungen eingebracht. Nun können vermehrt auch komplexe und multimodale Prozeduren abgebildet werden. Mit Hilfe dieser Diagnostik- und Behandlungskodes kann sichergestellt werden, dass die entsprechende DRG mit dem jeweiligen leistungsgerechten Kostengewicht angesteuert wird. Damit dient die ärztliche Dokumentation auch dazu, den Nachweis einer durchgeführten und möglicherweise kostenaufwendigen Behandlung im Rahmen einer Wirtschaftlichkeitsprüfung zu erbringen.

\section{Kodierung von Komplexprozeduren ist anspruchsvoll}

Die Erfassung ärztlicher Leistungen unter DRG stellt folgende Anforderungen: Sie soll zeitnah erfolgen und relevante diagnostische und therapeutische Massnahmen des aktuellen stationären Aufenthaltes vollständig abbilden. Und sie soll eine nachvollziehbare Kodierung auf der Grundlage gültiger Regelwerke und Kodierstandards ermöglichen.

Einfache Diagnostik- und Therapieverfahren können die Kodierfachpersonen leicht dem Austrittsbericht oder der Krankengeschichte entnehmen. Bei Komplexbehandlungen stellt sich die Situation jedoch etwas anders dar. Multimodale und komplexe Prozeduren zeichnen sich dadurch aus, dass sie bestimmte Inhalte oder Mindestmerkmale definieren. Erst die Erfüllung lässt die Vergabe des Kodes zu.

Dabei können die komplexen oder multimodalen, interdisziplinären Prozedurenkodes u.a. durch folgende Kriterien oder Mindestmerkmale definiert werden:
- Art/Anzahl der in die Behandlung eingebundenen Fachdisziplinen und Therapiebereiche

- Art/Anzahl von Therapieverfahren, die zum Einsatz kommen

- Staffelungen des Behandlungszeitraumes oder der Therapieeinheiten

Kurzum: Es handelt sich um eine Fülle von Einzelinformationen, die dem Behandelnden präsent sind, sich aber den in die Weiterbearbeitung der Daten involvierten Personen nur in entsprechend gebündelter Form erschliessen. Denn nur beim Vorliegen nachvollziehbarer Angaben kann der entsprechende CHOP-Kode verschlüsselt werden. Und nur die Kodes, die transparent und nachvollziehbar dokumentiert sind, werden Prüfungen von Kodierrevisoren oder Versicherungen standhalten. Fazit: Es braucht also eine Dokumentation, die aufzeigt, dass es sich um eine Komplexprozedur handelt.

\section{Dokumentationshilfen für komplexe Prozeduren}

Um den Ärzten Hilfe zur Erfassung komplexer Leistungen anbieten $\mathrm{zu}$ können, hat die FMH Dokumentations-Muster erarbeitet und sie den Fachgesellschaften zur Vernehmlassung vorgelegt. In diesen Mustern sind alle Informationen des betreffenden CHOP-Kodes übersichtlich aufbereitet und stehen den FMH-Mitgliedern unter www.fmh.ch $\rightarrow$ Tarife $\rightarrow$ SwissDRG $\rightarrow$ Ärztliche Dokumentation komplexer CHOP-Kodes zur Verfügung. Die Anwendung im Sinne einer Checkliste soll verhindern, dass durch das Dokumentieren komplexer Prozeduren ein unverhältnismässiger administrativer Mehraufwand entsteht. Die Dokumentations-Muster sind Vorschläge der FMH ohne jeglichen verbindlichen Charakter. Anpassungen an spitalinterne Bedürfnisse sind genauso möglich wie ein Einfügen in ein Krankenhaus-Informations-System. 